\title{
Philosophiques
}

\section{Remerciements à nos lecteurs et lectrices}

Volume 23, numéro 1, printemps 1996

Critères esthétiques et métamorphoses du beau

URI : https://id.erudit.org/iderudit/027393ar

DOI : https://doi.org/10.7202/027393ar

Aller au sommaire du numéro

Éditeur(s)

Société de philosophie du Québec

ISSN

0316-2923 (imprimé)

1492-1391 (numérique)

Découvrir la revue

Citer ce document

(1996). Remerciements à nos lecteurs et lectrices. Philosophiques, 23(1),

219-221. https://doi.org/10.7202/027393ar d'utilisation que vous pouvez consulter en ligne.

https://apropos.erudit.org/fr/usagers/politique-dutilisation/ 


\section{REMERCIEMENTS À NOS LECTEURS ET LECTRICES}

Depuis le $1^{\text {er }}$ aoūt 1993, les personnes suivantes ont accepté d'évaluer des textes pour Philosophiques. Nous les en remercions vivement.

AUMĖTRE, Jacques, UgAM, philosophie.

AYOUB, Josiane, UQAM, philosophie.

BÉGIN, Luc, UQAM, philosophie.

BOI, Luciano, UQAM, philosophie.

BOUCHARD, Guy, Université Laval, philosophie.

BLANCHARD, François, UGAM, philosophie.

BRUNELLE, Dorval, UQAM, sociologie.

CLOUTIER, Yvan, Collège de Sherbrooke, philosophie.

COUTURE, Jocelyne, UGAM, philosophie.

DE KONNINCK, Thomas, Université Laval, philosophie.

DESPRÉS, Pierre, Collège Montmorency, philosophie

DORION, Louis-André, Université de Montréal, philosophie.

DUCHASTEL, Jules, UgAM, sociologie.

DUCHESNEAU, François, Université de Montréal, philosophie.

DUMAS, Denis, Université d'Ottawa, philosophie.

DUMOUCHEL, Paul, UQAM, philosophie.

DUMOUCHEL, Daniel, Université de Montréal, philosophie.

FISETTE, Denis, UQAM, philosophie.

FOISY, Suzanne, UGTR, philosophie.

GAGNON, Maurice, Université de Sherbrooke, sciences humaines.

GAUTHIER, Yvon, Université de Montréal, philosophie.

GINGRAS, Yves, UGAM, histoire.

GRENON, Michel, UQAM, histoire.

GRONDIN, Jean, Université de Montréal, philosophie.

HJORT, Mette, Université McGill, Études françaises.

KAUFMANN, J. Nicolas, UGTR, philosophie.

KEMP, William, Collège du Vieux-Montréal, philosophie.

KNEE, Philip, Université Laval, philosophie.

LABERGE, Pierre, Université d'Ottawa, philosophie.

LACHANCE, Michael, UQAM, philosophie.

LACHARITÉ, Normand, UGAM, philosophie. 
LAFRANCE, Guy, Université d'Ottawa, philosophie.

LAFRANCE, Yvon, Université d'Ottawa, philosophie.

LAGUEUX, Maurice, Université de Montreal, philosophie.

LANGLOIS, Luc, Université Laval, philosophie.

LATRAVERSE, François, UQAM, philosophie.

LAMBERT, Roger, UQAM, philosophie.

LAURIER, Daniel, Université de Montréal, philosophie.

LAYWINE, Allison, Université McGill, philosophie.

LEBLANC, Hugues, UQAM, philosophie.

LEGAULT, Georges, Université de Sherbrooke, sciences humaines.

LEPAGE, François, Université de Montréal, philosophie.

LEROUX, Jean, Université d'Ottawa, philosophie.

LEROUX, Georges, UQAM, philosophie.

LETOCHA, Danièle, Université d'Ottawa, philosophie.

LEYDET, Dominique, UQAM, philosophie.

LÉVESGUE, Claude, Université de Montréal, philosophie

LIMOGES, Camille, UQAM, histoire.

LIVET, Pierre, Université de Provence, philosophie.

LIVINGSTONE, Paisley, Université McGill, anglais.

LUC, Laurent-Paul, Université de Sherbrooke, philosophie.

MALOUIN, Harel, UQAM, philosophie.

MARQUIS, Jean-Pierre, Université de Montréal, philosophie.

McCORMICK, Peter, Université d'Ottawa, philosophie.

MELLOS, Koula, Université d'Ottawa, sciences politiques.

MEUNIER, Jean-Guy, UGAM, philosophie.

MIGUELEZ, Roberto, Université d'Ottawa, sociologie.

MONETTE, Lise, UGAM, philosophie.

NADEAU, Robert, UQAM, philosophie.

NAUD, Julien, UQTR, philosophie.

NIELSEN, Kai, University of Calgary, philosophie.

PANACCIO, Claude, UQTR, philosophie.

PARADIS, André, UQTR, philosophie.

PICHÉ, Claude, Université de Montréal, philosophie.

QUINTIN, Paul-André, UQTR, philosophie.

ROBERT, Serge, UQAM, philosophie.

ROSS, Don, Université d'Ottawa, philosophie.

ROUSSEAU, Louis, UGAM, sciences de la religion.

ROY, Jean, Université de Montréal, philosophie.

RUELLAND, Jacques, Collège Édouard-Montpetit, philosophie.

SAUVE, Denis, CEGEP de Saint-Hyacinthe, philosophie.

SAVARY, Claude, UQTR, philosophie. 
SEYMOUR, Michel, Université de Montréal, philosophie. SIMARD, Jean-Claude, Collège de Rimouski, philosophie. SOSOÉ, Lukas, Université de Montréal, philosophie. TOURNIER, François, Université Laval, philosophie. TREPANIER, Esther, UQAM, histoire de l'art. VANDERVEKEN, Daniel, UGTR, philosophie. VERNES, Paule-Monique, Université de Provence, philosophie. VIDRICAIRE, André, UQAM, philosophie. VOIZARD, Alain, UGAM, philosophie. WEINSTOCK, Daniel, Université de Montréal, philosophie. Avril 1996. 